\title{
A Multivariate Analysis on the Effect of No Closed Suction Drain on the Length of Hospital Stay in Total Knee Arthroplasty
}

\author{
Kohei Nishitani, MD, Shinichi Kuriyama, MD, Shinichiro Nakamura, MD, Hiromu Ito, MD, and \\ Shuicih Matsuda, MD \\ Department of Orthopaedic Surgery, Graduate School of Medicine, Kyoto University, Kyoto, Japan
}

\begin{abstract}
Purpose: Despite the long history of drain use in total knee arthroplasty (TKA), no drain has been gaining popularity. The purpose of this study was to investigate whether drainage is related to the length of hospital stay.

Materials and Methods: A total of 166 consecutive unilateral TKAs performed on 135 patients with osteoarthritis were retrospectively reviewed. Closed suction drainage was used in 111 cases (67\%). Length of hospital stay after surgery was recorded, and a multivariate linear regression analysis was performed to evaluate various variables (patient factors, surgical factors, and post-surgical factors) and to investigate whether drainage was an independent variable.

Results: Hospital stay was shorter in no drain cases $(21.7 \pm 4.8$ days $)$ than in drain cases $(24.2 \pm 3.7$ days, $\mathrm{p}<0.001)$. The multivariate analysis showed that older age $(\beta=0.12, p=0.02)$, drain use $(\beta=2.81, p=0.03)$, and occurrence of comorbidity $(\beta=1.46, p=0.04)$ were the independent variables associated with the extended hospital stay. There was no difference in comorbidity between drain cases (39.6\%) and no drain cases (27.2\%, $\mathrm{p}=0.13)$. Conclusions: The drain use, age, and occurrence of comorbidity were related to the length of hospital stay. TKA without drain is an effective procedure both medically and economically.
\end{abstract}

Keywords: Knee, Arthroplasty, Drain, Hospitalization, Recovery

\section{Introduction}

Total knee arthroplasty (TKA) has been the most reliable treatment for end-stage osteoarthritis to relieve pain and improve function. Closed suction drainage (CSD) has been the standard procedure in TKA ${ }^{1)}$. CSD is used to decrease hematoma formation and intraarticular pressure that cause wound leakage, ecchy-

Received February 5, 2018; Revised (1st) May 20, 2018;

(2nd) October 4, 2018; Accepted October 4, 2018

Correspondence to: Kohei Nishitani, MD

Department of Orthopaedic Surgery, Graduate School of Medicine, Kyoto University, 54 Shogoin-Kawahara-Cho, Sakyo, Kyoto 606-8507, Japan

Tel: +81-75-751-3366, Fax: +81-75-751-8409

E-mail: nkohei@kuhp.kyoto-u.ac.jp

This is an Open Access article distributed under the terms of the Creative Commons Attribution Non-Commercial License (http://creativecommons.org/licenses/by-nc/4.0/) which permits unrestricted non-commercial use, distribution, and reproduction in any medium, provided the original work is properly cited. mosis, and postoperative inflammation, leading to loss of range of motion (ROM) and delayed postoperative recovery ${ }^{2,3)}$. In addition, hematoma is thought to serve as a good broth for bacteria, and less hematoma formation is believed to decrease unpleasant postoperative implant-associated infection ${ }^{2,3)}$.

However, the drain tube can provide a route of retrograde infection and may inhibit early ambulation and commencement of postoperative rehabilitation ${ }^{3)}$. Recently, a trend of no drain has been gaining popularity on the basis of no differences in infection rates, pain, and functional recovery ${ }^{3,4}$. Several reports have even described decreased postoperative blood loss and blood transfusion $^{4,5)}$. As described above, the advantages and disadvantages of the use of drain for TKA are still controversial. Although recent meta-analyses failed to show effects of drain use on hospital stay $^{6}$, some recent articles described that drain use was related to prolonged hospital stay ${ }^{7,8)}$.

Together with the drain use, the use of tranexamic acid and multimodal analgesic cocktails may influence the length of hos- 
pital stay. The use of tranexamic acid decreases hematoma formation and blood loss. A recent meta-analysis reported that the use of tranexamic acid was related to a shorter length of hospital stay $^{9)}$. The use of a multimodal analgesic cocktail gives effective pain relief and functional recovery, which may also shorten the hospital stay ${ }^{10)}$.

In this article, we evaluated the effects of drain use and other perioperative procedures, such as the use of tranexamic acid, multimodal analgesic cocktails, and anticoagulant therapy, on hospital stay in a university hospital in Japan. The hypothesis of this study was that no drain use was related to a shortened hospital stay.

Table 1. Characteristics of Knee Osteoarthritis Patients

\begin{tabular}{lcccc}
\hline \multicolumn{1}{c}{ Characteristic } & Total & No drain & $\begin{array}{c}\text { Closed } \\
\text { suction } \\
\text { drainage }\end{array}$ & p-value \\
\hline No. of knees (patient) & $166(135)$ & $55(52)$ & $111(94)$ & - \\
Age (yr) & $74.5 \pm 7.5$ & $74.3 \pm 8.7$ & $74.6 \pm 7.2$ & 0.428 \\
Sex (male:female) & $31: 135$ & $18: 42$ & $13: 93$ & 0.007 \\
Right:left & $86: 80$ & $29: 26$ & $57: 54$ & 0.999 \\
Body mass index & $27.7 \pm 4.7$ & $26.5 \pm 4.3$ & $26.9 \pm 5.0$ & 0.584 \\
$\quad$ (kg/m ${ }^{2}$ ) & & & & \\
Operation time (min) & $113.3 \pm 33.0$ & $103.7 \pm 27.2$ & $118.1 \pm 34.2$ & 0.007 \\
Implant (CR/CS/PS) & $28 / 125 / 13$ & $11 / 36 / 8$ & $17 / 89 / 5$ & 0.051 \\
Tranexamic acid & $133(80)$ & $55(100)$ & $78(70)$ & $<0.001$ \\
Multimodal analgesic & $54(33)$ & $47(85)$ & $8(7)$ & $<0.001$ \\
$\quad$ cocktail & & & & \\
Anticoagulant & $78(47)$ & $14(25)$ & $64(58)$ & $<0.001$ \\
Comorbidity & $59(35)$ & $15(27)$ & $44(39)$ & 0.243 \\
\hline
\end{tabular}

Values are presented as mean \pm standard deviation or number (\%). CR: cruciate-retaining, CS: cruciate-substituting, PS: posterior-stabilizing.

\section{Materials and Methods}

This study was approved by the Ethics Committee of our hospital, and written informed consents were obtained from all participants. One-hundred and sixty-six unilateral consecutive primary TKAs performed on 135 patients with osteoarthritis between April 2012 and March 2015 were included in this retrospective study (Table 1). TKA was performed by six boardcertificated orthopedic surgeons with the patient under general anesthesia through a midline skin incision and medial parapatellar approach using the conventional cement technique. Among the 166 TKAs, a cruciate-retaining (CR) prosthesis (NexGen CRFlex; Zimmer Inc., Warsaw, IN, USA) was implanted in 28 knees, a cruciate-substituting (CS) prosthesis was used in 125 knees (BiSurface Knee System; Kyocera, Kyoto, Japan), and a posteriorstabilizing (PS) prosthesis (NexGen LPS-Flex, Zimmer Inc.) was used in 13 knees. In the early study period, CSD was used in all cases. Thereafter, the use of tranexamic acid, a multimodal analgesic cocktail, or CSD was determined according to the surgeon's preference. In case of tranexamic acid used with CSD, the drain was clamped for 2 hours. For TKAs performed in the late study period, tranexamic acid and a multimodal analgesic cocktail were used without a drain. The bar chart in Fig. 1 shows the percentage of each procedure in each year. For the prophylaxis of postoperative deep vein thrombosis (DVT), intermittent pneumatic compression was routinely used in all cases, unless contraindication was present. However, the use of anticoagulants was dependent on the risk involved in each case. CSD was routinely removed 2 days after surgery. In no drain cases, ambulation was permitted on postoperative day 1 at the earliest, but ambulation was allowed as early as 2 days postoperatively in CSD cases. Continuous passive motion was commenced on postoperative day 2 in no drain cases and on day 2 or 3 in CSD cases. Full weight bearing was allowed immediately after ambulation, and ROM
A

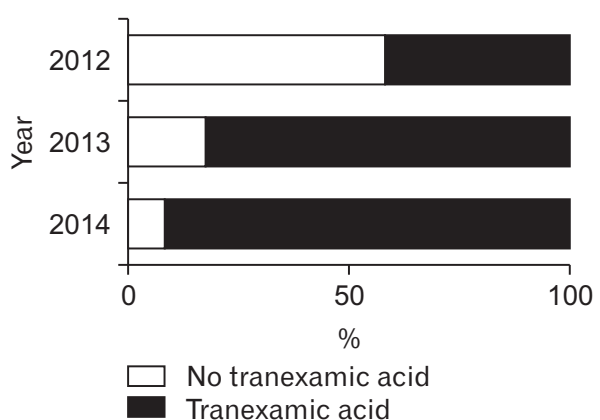

$\mathrm{B}$

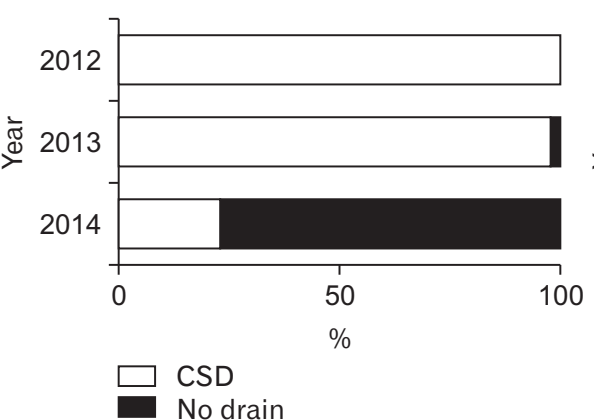

C

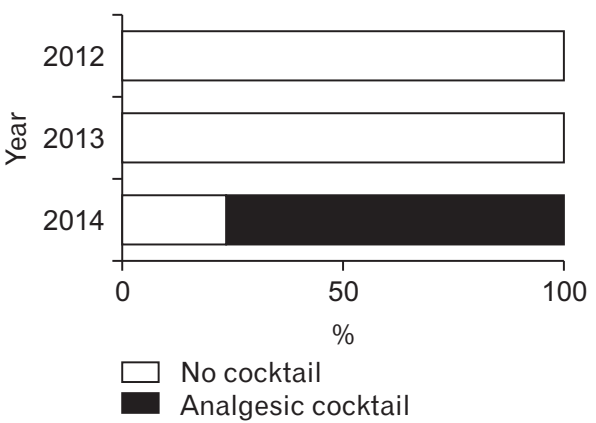

Fig. 1. Bar charts showing frequency of each procedure in each year. (A) Use of tranexamic acid. (B) Use of drain. (C) Use of an analgesic cocktail. CSD: closed suction drainage. 
and functional exercises were supervised by a physiotherapist. The length of hospital stay after surgery was recorded in all cases. Any comorbidity after surgery was recorded, including deep or superficial infection, DVT, pulmonary embolism, heart, gastric, liver or renal problem, psychic disorder, such as delirium or dementia, and blood transfusion. To predict blood loss, peripheral blood hemoglobin was recorded preoperatively and postoperatively. Among the postoperative hemoglobin levels within 7 days, the lowest level of hemoglobin was chosen to calculate a decrease in hemoglobin (preoperative hemoglobin-postoperative minimum hemoglobin). At discharge, the Timed Up and Go (TUG) test was performed to evaluate functional recovery, as previously described ${ }^{11)}$, and the strength of knee extension was recorded with the CYBEX HUMAC NORM (Computer Sports Medicine Inc., Stoughton, MA, USA) dynamometer to evaluate recovery of quadriceps strength $^{11}$.

\section{Statistical Analysis}

The Student $t$-test was used to compare CSD and no drain cases. To investigate the dependent variables of hospital stay, multivariate linear regression analysis was performed. To include the yearly bias in the analysis, the duration from April 2012 to March 2013 (2012 fiscal and school year) was defined as the first year, the duration from April 2013 to March 2014 (2013 fiscal and school year) was defined as the second year, and the duration from April 2014 to March 2015 (2014 fiscal and school year) was defined as the third year; 1, 2, or 3 was substituted accordingly in the multivariate analysis. For the implant design, we defined $\mathrm{CR}=1, \mathrm{CS}=2$, and $\mathrm{PS}=3$ since the $\mathrm{CR}$ implant was used for moderate deformity, PS implant was used for advanced deformity, and the CS implant was used for all others. The occurrence of comorbidity, wound problem, and blood transfusion was compared between CSD and no drain cases with Fisher exact test. A p-value of less than 0.05 was considered significant.

\section{Results}

Hospital stay was shorter in no drain cases $(21.7 \pm 4.8$ days) than in CSD cases (24.2 \pm 3.7 days, $\mathrm{p}<0.001)$ (Fig. 2). Hospital stay was $23.4 \pm 2.8$ days during the first year, $25.0 \pm 2.8$ days during the second year, and $22.1 \pm 4.4$ days during the third year. There was statistical difference only between the second and the third year $(p<0.001)$, but there was no difference between the first and the third year $(\mathrm{p}=0.15)$. Due to the high heterogeneity of the patient population between two groups (Table 1), the difference of hospital stay could also be affected by other variables. Thus, multivari-

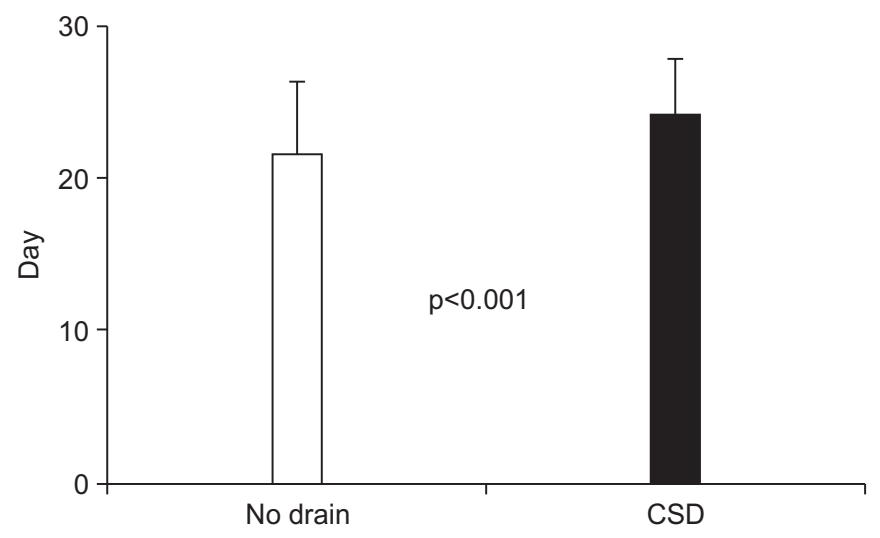

Fig. 2. Hospital stay of no drain cases and closed suction drainage (CSD) cases. Data are shown as mean \pm standard deviation.

ate analysis was performed to know if drain use was the independent factor that influences hospital stay (Table 2). The use of the CSD, occurrence of comorbidity, and older age were found to be the independent predictors of longer hospital stay. A strong knee extensor was nearly related to a shorter hospital stay $(\mathrm{p}=0.06)$, although it was not significant. The operation year had no effect on the length of hospital stay in this multivariate regression model.

There was no difference in the occurrence of comorbidities between no drain (15/55 cases, 27.2\%) and CSD cases (44/111 cases, 39.6\%). There was no deep infection throughout the study period. Four wound problems were observed in no drain cases, while 2 were observed in CSD cases $(\mathrm{p}=0.09)$, and 1 no drain case needed debridement and additional sutures. In no drain cases, less hemoglobin was lost (decrease of hemoglobin, 2.1 \pm 1.0 $\mathrm{mg} / \mathrm{dL}$ ) compared to CSD cases (decrease of hemoglobin, 2.6 \pm 1.2 $\mathrm{mg} / \mathrm{dL} ; \mathrm{p}=0.009)$. Blood transfusion was needed in $5 \mathrm{CSD}$ cases (4.5\%) and 1 no drain case (1.8\%); however, there was no statistical difference $(\mathrm{p}=0.66)$.

No drain cases showed better recovery of function. Knee extension strength was not different between no drain $(0.66 \pm 0.36 \mathrm{Nm} /$ $\mathrm{kg}$ ) and CSD cases $(0.60 \pm 0.31 \mathrm{Nm} / \mathrm{kg})$ (Fig. 3A). However, no drain cases could complete the TUG test in a shorter period of time (10.8 \pm 3.7 seconds) than CSD cases (13.6 \pm 7.2 seconds) at discharge (Fig. 3B).

\section{Discussion}

In the current study, the use of CSD was correlated with a longer hospital stay after TKA. The strength of this study was in the multivariate analysis using most variables that could affect the length of hospital stay, in which the use of CSD was an independent predictor of a longer hospital stay together with the occur- 
Table 2. Multivariate Regression Analysis for the Length of Hospital Stay

\begin{tabular}{|c|c|c|c|c|}
\hline Characteristic & Substitute value & $\beta(95 \% \mathrm{CI})$ & t-value & $\mathrm{p}$-value \\
\hline Age (yr) & & $0.12(0.02$ to 0.21$)$ & 2.47 & 0.02 \\
\hline \multicolumn{5}{|l|}{ Sex } \\
\hline Male/female & $1 / 0$ & $0.06(-1.81$ to 1.92$)$ & 0.06 & 0.95 \\
\hline Body mass index $\left(\mathrm{kg} / \mathrm{m}^{2}\right)$ & & $-0.01(-0.16$ to 0.15$)$ & -0.10 & 0.92 \\
\hline Knee extension strength $(\mathrm{Nm} / \mathrm{kg})$ & & $-1.70(-3.47$ to 0.07$)$ & -1.90 & 0.06 \\
\hline \multicolumn{5}{|l|}{ Operation year } \\
\hline April 2012-March 2013 & 1 & $0.83(-0.49$ to 2.14$)$ & 1.25 & 0.21 \\
\hline April 2013-March 2014 & 2 & & & \\
\hline April 2014-March 2015 & 3 & & & \\
\hline Operation time (min) & & $0.00(-0.02$ to 0.03$)$ & 0.26 & 0.80 \\
\hline \multicolumn{5}{|l|}{ Implant design } \\
\hline $\mathrm{CR} / \mathrm{CS} / \mathrm{PS}$ & $1 / 2 / 3$ & $0.65(-0.68$ to 1.99$)$ & 0.97 & 0.33 \\
\hline \multicolumn{5}{|l|}{ Closed suction drainage } \\
\hline No use/use & $0 / 1$ & $2.81(0.22$ to 5.4$)$ & 2.14 & 0.03 \\
\hline \multicolumn{5}{|l|}{ Tranexamic acid } \\
\hline No use/use & $0 / 1$ & $-0.70(-2.71$ to 1.31$)$ & -0.69 & 0.49 \\
\hline \multicolumn{5}{|l|}{ Multimodal analgesic cocktail } \\
\hline No use/use & $0 / 1$ & $0.34(-2.19$ to 2.87$)$ & 0.27 & 0.79 \\
\hline \multicolumn{5}{|l|}{ Anticoagulant } \\
\hline No use/use & $0 / 1$ & $-0.06(-1.55$ to 1.42$)$ & -0.09 & 0.93 \\
\hline \multicolumn{5}{|l|}{ Comorbidity } \\
\hline No occurrence/occurrence & $0 / 1$ & $1.46(0.06$ to 2.86$)$ & 2.06 & 0.04 \\
\hline
\end{tabular}

CI: confidence interval, CR: cruciate-retaining, CS: cruciate-substituting, PS: posterior-stabilizing.
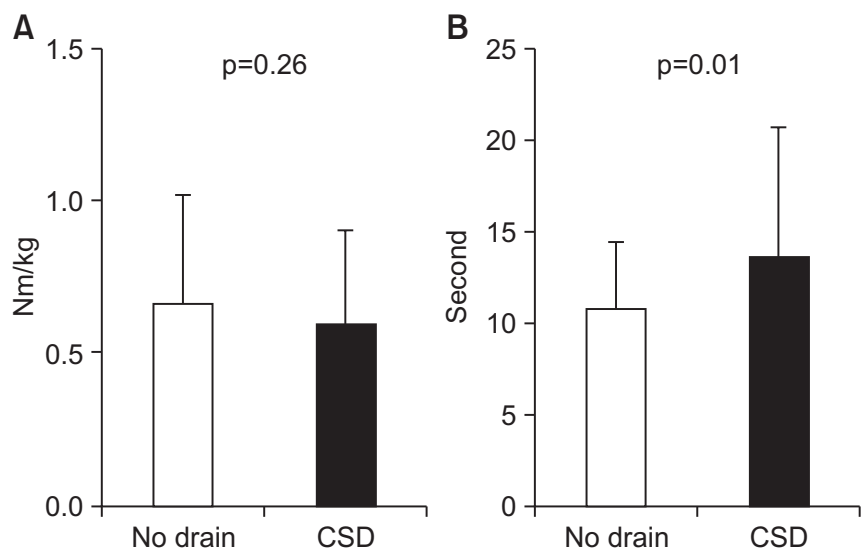

Fig. 3. Functional recovery at discharge. (A) Knee extension strength. (B) Timed Up and Go test. CSD: closed suction drainage.

rence of comorbidity and older age of the patient.

In a recent meta-analysis published in 2016, Hong et al. ${ }^{6}$ reported that no drain use showed no favorable trend compared to autologous blood transfusion drainage system. In their analysis, only 2 articles had no drain use group, and there was no comparison between no drain use and CSD. Other than this meta-analysis, there are 3 recent articles comparing no drain use and CSD. Wang et al. ${ }^{8)}$ concluded that TKA without CSD was associated with faster recovery, better function, and 0.9 days shorter hospital stay (7.1 \pm 1.9 days in CSD vs. $6.2 \pm 1.9$ days in non-CSD) in their randomized control trial including 80 patients. In a retrospective observational study, Chen et al. ${ }^{12)}$ described no difference in the length of hospital stay between drain use (median length of stay, 4 days; interquartile range, 4 to 5 days) and no drain use (median length of stay, 4 days; interquartile range, 3 to 5 days) ( $\mathrm{p}=0.228$ ). On the other hand, Sharma et al. reported a shorter hospital stay in the no drain group $(5.12 \pm 1.65$ days) than in the drain group $(6.21 \pm 1.74 \text { days, } \mathrm{p}<0.001)^{7}$. The health care system differs among countries, which also affects the hospital stay. These studies showed the effect of no drain use on shorter hospital stay in the very early recovery period (less than 1 week), whereas our study showed the effect of no drain use at about 3 weeks after surgery. There was no early readmission after TKA in this study, although 
Chen et al ${ }^{12)}$ reported 30-day readmission in a small percent of patients.

Two previous meta-analyses showed that CSD reduced wound problems, though a recent meta-analysis showed no difference in wound infection. Several studies reported an increase in wound problems and ecchymosis when drain was not used ${ }^{2}$, and a trend of more wound problems $(\mathrm{p}=0.09)$ was also seen in the current study. Regarding wound healing, reduced swelling, and inflammation, CSD may have some advantages. Deep infection is one of the worst complications of TKA. Theoretically, hematoma formation and tissue damage by high pressure are the risk factors for deep-seated infection, but the drain lumen also provides a route for retrograde migration of bacteria ${ }^{13)}$. A meta-analysis of 36 studies, involving 5,464 participants undergoing different types of orthopedic surgery, did not find difference in the infection rate following the drain use ${ }^{14}$. In our study, no deep infection was observed. However, due to the limited number of patients, the effect of CSD on deep infection could not be determined.

Some studies reported that the drain use increases blood $\operatorname{loss}^{4,5,12)}$, whereas others reported no such relationship ${ }^{15,16}$. In this study, the decrease in the hemoglobin level was more pronounced following CSD. However, the use of drain clamping, tranexamic acid, multimodal analgesic cocktails, and postoperative anti-coagulants may largely affect the results. Thus, we cannot conclude the effect of not using CSD on blood loss prevention after TKA.

In this study, no drain cases showed better function than CSD cases in some aspect. Wang et al. ${ }^{8}$ reported better ROM following no drain use, whereas others reported no such difference ${ }^{3)}$. In the patient outcome report, no drain use did not affect the Western Ontario and McMaster Universities Osteoarthritis Index or Oxford Knee Score ${ }^{7,8)}$. In our report, no drain cases showed better TUG test at discharge, which is a useful tool for quantifying function of mobility based on its validity and reliability ${ }^{17)}$. However, other procedures such as analgesic cocktails could have influence on the TUG test due to the heterogeneity of the patient population, and we cannot conclude that no drain contributed to the functional recovery.

The current article has some limitations. First, this is a retrospective observational study with a limited number of patients. A prospective study would be ideal to obtain strong evidence of relationship between the CSD use and the length of hospital stay. Second, there was high heterogeneity in perioperative procedures, which were chosen by the surgeons. However, to identify the independent effect of drain on hospital stay, multivariate analysis was conducted including all the procedures, and the use of CSD was still an independent predictor of the longer hospital stay. Third, only CSD was compared with no drain. The relative advantage of autologous blood transfusion drainage on the length of hospital stay or total blood loss over CSD has been reported ${ }^{6}$. A further study is needed to determine the advantage of no drain against autologous blood transfusion drain. Fourth, only patients with osteoarthritis were included, and the effect of no drain on systematic arthritis, such as rheumatoid arthritis, remains unknown. Fifth, the length of hospital stay was as long as 23.6 days and longer than other reports. However, the hospitalization period in this study was shorter than that in previous studies that showed more than 1 month of hospital stay ${ }^{18,19)}$ and similar to a recent study in which the hospital stay was approximately 24 days $^{20}$. Sixth, as described in the Method section, the commencement of ambulation was delayed in CSD cases; the effect of the delay on the length of hospital stay could not be excluded.

\section{Conclusions}

The multivariate regression analysis showed the use of CSD was independently related to a longer hospital stay, together with older age and occurrence of comorbidities. Although the use of drain has a long history in TKA, TKA without drainage can be considered a treatment option for medical and economic reasons.

\section{Conflict of Interest}

No potential conflict of interest relevant to this article was reported.

\section{References}

1. Waugh TR, Stinchfield FE. Suction drainage of orthopaedic wounds. J Bone Joint Surg Am. 1961;43:939-46.

2. Kim YH, Cho SH, Kim RS. Drainage versus nondrainage in simultaneous bilateral total hip arthroplasties. J Arthroplasty. 1998;13:156-61.

3. Esler CN, Blakeway C, Fiddian NJ. The use of a closedsuction drain in total knee arthroplasty. A prospective, randomised study. J Bone Joint Surg Br. 2003;85:215-7.

4. Walmsley PJ, Kelly MB, Hill RM, Brenkel I. A prospective, randomised, controlled trial of the use of drains in total hip arthroplasty. J Bone Joint Surg Br. 2005;87:1397-401.

5. Holt JB, Miller BJ, Callaghan JJ, Clark CR, Willenborg MD, Noiseux NO. Minimizing blood transfusion in total hip and knee arthroplasty through a multimodal approach. J Arthroplasty. 2016;31:378-82. 
6. Hong KH, Pan JK, Yang WY, Luo MH, Xu SC, Liu J. Comparison between autologous blood transfusion drainage and closed-suction drainage/no drainage in total knee arthroplasty: a meta-analysis. BMC Musculoskelet Disord. 2016; 17:142.

7. Sharma GM, Palekar G, Tanna DD. Use of closed suction drain after primary total knee arthroplasty - an overrated practice. SICOT J. 2016;2:39.

8. Wang D, Xu J, Zeng WN, Zhou K, Xie TH, Chen Z, Yu HD, Li JL, Zhou ZK, Pei FX. Closed suction drainage is not associated with faster recovery after total knee arthroplasty: a prospective randomized controlled study of 80 patients. Orthop Surg. 2016;8:226-33.

9. Tian P, Liu WB, Li ZJ, Xu GJ, Huang YT, Ma XL. The efficacy and safety of tranexamic acid in revision total knee arthroplasty: a meta-analysis. BMC Musculoskelet Disord. 2017;18:273

10. Lee YS. Comprehensive analysis of pain management after total knee arthroplasty. Knee Surg Relat Res. 2017;29:80-6.

11. Furu M, Ito H, Nishikawa T, Nankaku M, Kuriyama S, Ishikawa M, Nakamura S, Azukizawa M, Hamamoto Y, Matsuda S. Quadriceps strength affects patient satisfaction after total knee arthroplasty. J Orthop Sci. 2016;21:38-43.

12. Chen JY, Lee WC, Chan HY, Chang PC, Lo NN, Yeo SJ. Drain use in total knee arthroplasty is neither associated with a greater transfusion rate nor a longer hospital stay. Int Orthop. 2016;40:2505-9.

13. Saleh K, Olson M, Resig S, Bershadsky B, Kuskowski M,
Gioe T, Robinson H, Schmidt R, McElfresh E. Predictors of wound infection in hip and knee joint replacement: results from a 20 year surveillance program. J Orthop Res. 2002;20: 506-15.

14. Parker MJ, Livingstone V, Clifton R, McKee A. Closed suction surgical wound drainage after orthopaedic surgery. Cochrane Database Syst Rev. 2007;(3):CD001825.

15. Crevoisier XM, Reber P, Noesberger B. Is suction drainage necessary after total joint arthroplasty? A prospective study. Arch Orthop Trauma Surg. 1998;117:121-4.

16. Sundaram RO, Parkinson RW. Closed suction drains do not increase the blood transfusion rates in patients undergoing total knee arthroplasty. Int Orthop. 2007;31:613-6.

17. Podsiadlo D, Richardson S. The timed "Up \& Go": a test of basic functional mobility for frail elderly persons. J Am Geriatr Soc. 1991;39:142-8.

18. Yasunaga H, Tsuchiya K, Matsuyama Y, Ohe K. Analysis of factors affecting operating time, postoperative complications, and length of stay for total knee arthroplasty: nationwide web-based survey. J Orthop Sci. 2009;14:10-6.

19. Ishii Y, Noguchi H, Takeda M, Sato J, Suzuki T. Length of hospital stay with patient-dependent determination in bilateral scheduled staged total knee arthroplasty. Eur J Orthop Surg Traumatol. 2014;24:961-5.

20. Tanikawa H, Harato K, Ogawa R, Sato T, Kobayashi S, Nomoto S, Niki Y, Okuma K. Local infiltration of analgesia and sciatic nerve block provide similar pain relief after total knee arthroplasty. J Orthop Surg Res. 2017;12:109. 